\title{
Multi-objective Parameters Optimization for HEV Based on improved Particle Swarm Algorithm
}

\author{
Ying $\mathrm{Ai}^{1, *}$, Yuanjie $\mathrm{Gao}^{2}$, and dongsheng $\mathrm{Liu}^{3}$ \\ ${ }^{1}$ Wuhan donghu new technology development zone power supply company \\ ${ }^{2}$ Hubei power company material company \\ ${ }^{3}$ Wuhan Product Quality Supervision and Inspection Institute
}

\begin{abstract}
Hybrid electric vehicle fuel consumption and emissions are closely related to its energy management strategy. A fuzzy controller of energy management using vehicle torque request and battery state of charge (SOC) as inputs, engine torque as output is designed in this paper foe parallel hybrid electric vehicle. And a multi-objective mathematical function which purpose on maximize fuel economy and minimize emissions is also established, in order to improve the adaptive ability and the control precision of basic fuzzy controller, this paper proposed an improved particle swarm algorithm that based on dynamic learning factor and adaptive inertia weight to optimize the control parameters. Simulation results based on ADVISOR software platform show that the optimized energy management strategy has a better distribution of engine and motor torque, which helps to improved the vehicle's fuel economy and exhaust emission performance.
\end{abstract}

\section{Introduction}

Parallel hybrid electric vehicle(PHEV) [1] is a kind of vehicle that mainly driven by engine and motor-driven as auxiliary, it has the characteristics of flexible control and high comprehensive efficiency, but due to the complexity of its running model, it becomes necessary to develop a reasonable torque distribution scheme, so as to realize the energy distribute effectively between engine and motor, which can help vehicle to obtain the maximum fuel economy, lowest emissions and steady performance of the vehicle under the premise of meet its dynamic performance. Fuzzy logic control [2] has the characteristics of strong robustness, good real-time and strong logicality,etc, now it has been widely applied to the control of HEV. The effect of fuzzy logic control is closely related to parameters' selection of membership function, however this process is usually done according to experts' experience in practical application, so its control precision still remains to be optimized. Particle swarm algorithm [3] has the advantages of high search efficiency, easy to realize, and less parameters to adjust in the same kind of intelligent optimization algorithms, such as genetic algorithm [4], simulated annealing algorithm[5] and other evolutionary algorithms, which make it suitable for parameter optimization of hybrid cars. Aimed at parallel hybrid electric vehicle, this paper designed a fuzzy controller by using vehicle torque request and battery SOC as inputs, and engine torque as output, then adopting improved particle swarm algorithm to optimize the control parameters, simulation results show that the optimized fuzzy energy management strategy can more effectively improve the vehicle fuel economy and emission performance.

\section{Fuzzy control strategy}

The principle diagram of the double inputs and single output fuzzy torque controller by using vehicle torque request and battery SOC as inputs, engine torque as output as it shown in figure 1.

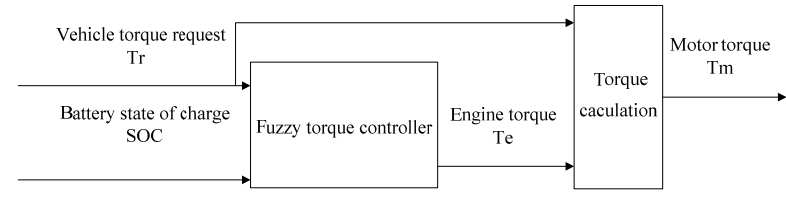

Fig. 1. Fuzzy torque control principle diagram.

The parameters selection of fuzzy membership function determine the control precision of the controller, this paper uses the overlapping symmetrical triangle and trapezoid as the membership function of each parameter variable. Among them, all domain of the input and output variables are set in $[0,1]$, the two input variables are divided into five fuzzy subset, namely\{VS, S, M, B, $\mathrm{VB}$ \}, as it shown in figure 2 (a); the output variables are divided into seven fuzzy subset, namely $\{\mathrm{VS}, \mathrm{S}, \mathrm{TS}, \mathrm{M}$, $\mathrm{TB}, \mathrm{B}, \mathrm{VB}\}$, as it shown in figure 2 (b). The scope of all control variables is $[0,0.4]$. 


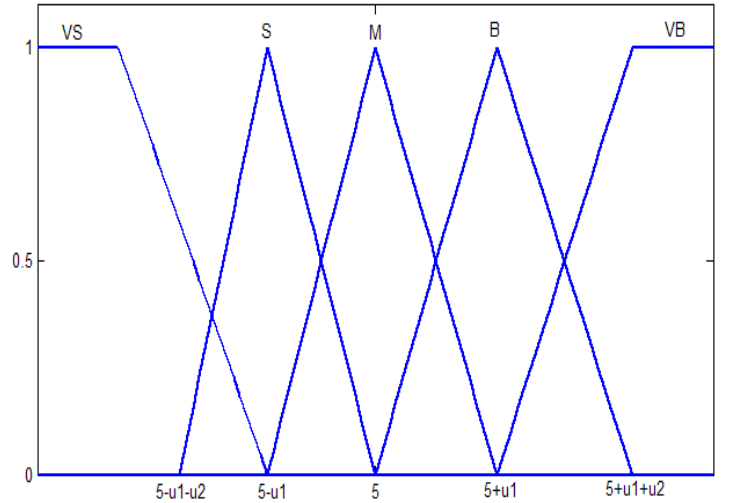

(a)Parameters of input membership functions

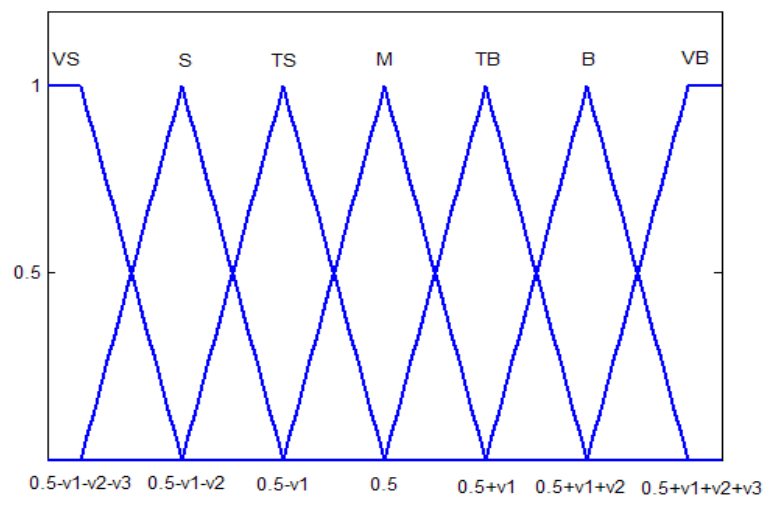

(b)Parameters of output membership functions

Fig. 2. Membership function

\subsection{Improved particle swarm algorithm}

Particle swarm algorithm is a heuristic algorithm based on multi-point random search, each particle is composed of two vector: position vector and the velocity vector. The position of particle $\mathrm{i}$ in $\mathrm{N}$ dimensional space is $X_{i}=\left(x_{i 1}, x_{i 2}, \ldots, x_{i N}\right)$, flight speed is $V_{i}=\left(v_{i 1}, v_{i 2}, \ldots, v_{i N}\right)$, each particle search for their individual extremum $P_{b e s t i}$ and global extremum $G_{\text {bestk }}$ by exchanging and sharing the information among the groups, then quickly converge to a point in the solution space namely the optimal solution. Particles update their speed and position according to the following formula :

$$
\begin{aligned}
& v_{i, d}^{(k+1)}=\omega v_{i, d}^{(k)}+c_{1} r_{1}\left[p_{i, d}^{(k)}-x_{i, d}^{(k)}\right]+c_{2} r_{2}\left[g_{i, d}^{(k)}-x_{i, d}^{(k)}\right] \\
& x_{i, d}^{(k+1)}=x_{i, d}^{(k)}+v_{i, d}^{(k+1)}
\end{aligned}
$$

In the type: $k$ is iteration number; $\omega$ is the inertia weigh coefficient; $c^{c_{1}}$ and $c_{2}$ are the acceleration weight coefficient; $r_{1}$ and $r_{2}$ are random numbers which are distributed evenly between 0 and $1 ; v_{i, d}^{(k)}$ is the velocity of particle ${ }^{i} ; x_{i, d}^{(k)}$ is the position of particle ${ }^{i}$.

In the process of particle swarm algorithm search, it is often hoped that particles have strong global search ability in early iteration, and strong local search ability in late iteration, so as to avoid premature convergence and improve its solution accuracy. To this end, this paper introduced the dynamic learning factor and adaptive inertia weight these two strategy to improve its convergence speed and optimization precision.

\subsection{Dynamically adjusting of learning factor}

Learning factors are the parameters that used to change the particle' s personal experience and groups' social experience in the searching process, reflects the information exchange between particles. Dynamically adjust the learning factor formula is as follows :

$$
\left\{\begin{array}{l}
c_{1}=2 \sin ^{2}\left[\frac{\pi}{2}\left(1-\frac{k}{t_{\max }}\right)\right] \\
c_{2}=2 \sin ^{2}\left(\frac{\pi k}{2 t_{\max }}\right)
\end{array}\right.
$$

In the type(3): ${ }^{c_{1}}$ and ${ }^{c_{2}}$ are monotone decreasing and increasing function of $k$ respectively. ${ }^{c_{1}}$ has larger value and $c_{2}$ has smaller value in early iteration, which can enlarge their search scope, improve particles' global search ability; in late iteration ${ }^{c_{1}}$ has smaller value and $c_{2}$ has larger value, so as to improve their local search ability and converge to global optimal solution quickly.

\subsection{Dynamically adjusting of inertia weight}

The single adjustment of learning factors is beneficial to improve the convergence speed of the algorithm, but its optimization precision remains to be improved, this paper use adjust inertia weight method based on particle individual fitness value, so as to maintain the group diversity better, then further to improve the convergence precision of the algorithm. formula is as follows:

$\omega= \begin{cases}\omega_{\min }+\frac{\left(\omega_{\max }-\omega_{\min }\right)\left(f_{i}-f_{\min }\right)}{f_{a v}-f_{\min }} & , f_{i} \leq f_{a v} \\ \omega_{\max } & , f_{i}>f_{a v}\end{cases}$

In the type(4): $\omega_{\max }$ and $\omega_{\min }$ is the maximum and minimum value of $\omega$ respectively; $f_{i}$ is the fitness value of particle $i, f_{\text {av }}$ is the average fitness value of the group.

\subsection{Objective Function}

Aimed at minimizing the vehicle fuel consumption and emissions under the premise of satisfy the vehicle dynamic performance, the multi-objective function is established in this paper as follows:

$$
\left\{\begin{array}{lr}
\min F(\mathrm{X})=\left[F C(\mathrm{X}), C O(\mathrm{X}), H C(\mathrm{X}), N O_{x}(\mathrm{X})\right] \\
\text { s.t. } \quad v_{i}(\mathrm{X})>0 & i=1,2, \ldots n
\end{array}\right.
$$


In the type(5): $X$ is parameter vector; $F C(\mathrm{X})$ is fuel consumption; $C O(\mathrm{X}), H C(\mathrm{X})$ and ${ }^{N O_{x}(\mathrm{X})}$ are the emissions of $C O, H C$ and $N O_{X}$ respectively; $v_{i}(\mathrm{X})>0$ is the highest speed, climbing ability constraints, etc that the car must satisfied.Weighting method is used to change the multiobjective optimization problem into a single objective problem as follows:

$\min F(\mathrm{X})=\lambda_{1} F C(\mathrm{X})+\lambda_{2} C O(\mathrm{X})+\lambda_{3} H C(\mathrm{X})+\lambda_{4} N O_{x}(\mathrm{X})$

In the type(6): $\lambda_{1}, \lambda_{2}, \lambda_{3}$ and $\lambda_{4}$ are the weight coefficient of each target and content in this paper $\lambda_{1}+\lambda_{2}+\lambda_{3}+\lambda_{4}=1, \lambda_{1}=0.4, \lambda_{2}=\lambda_{3}=\lambda_{4}=0.2$

\subsection{Basic steps of improved particle swarm algorithm for multi-objective parameter optimization}

- Parameter initialization: $\omega_{\max }=0.9, \omega_{\min }=0.4$; initial population $S=15$; $\max$ iterations $\mathrm{t}_{\max }=30$; particle dimension $D=7$; encode each parameter of input and output membership functions.Avoid combining SI and CGS units, such as current in amperes and magnetic field in oersteds. This often leads to confusion because equations do not balance dimensionally. If you must use mixed units, clearly state the units for each quantity that you use in an equation.

- Fitness calculation: each particle's fitness value should be calculated snd compared according to (6), which can help to update the individual optimal $P_{\text {besti }}$ and global optimal $G_{\text {bestk }}$.

- Parameter adjustment: adjust learning factor and weight coefficient according to (3) and (4), then update their speed and position according to (1) and (2).

- The algorithm should be determined that whether it meet the termination conditions. If the maximum number of iterations, putout the optimal solution; otherwise, return to step (2) and continue the iterative calculation.

\section{Simulation analysis}

This paper proposed to use improved particle swarm algorithm to optimize energy management strategy based on fuzzy control, firstly write the corresponding simulation program in the MATLAB6.1 environment, optimize parameters of membership functions by calling related data in ADVISOR2002; then test in three typical driving cycles CYC_NEDC, CYC_UDDS, CYC_1015, and get engine optimal working point under different working conditions; compare the fuel economy and emissions of hybrid electric vehicle based on fuzzy control before and after optimization, simulation results are shown in table 2:Equations should be centred and should be numbered with the number on the right-hand side.

Table 1. Comparison of simulation results for three kinds of typical road conditions

\begin{tabular}{ccccc}
\hline \multirow{2}{*}{$\begin{array}{c}\text { Control } \\
\text { strategy }\end{array}$} & \multicolumn{4}{c}{ CYC-NEDC } \\
\cline { 2 - 5 } & $\mathrm{HC} / \mathrm{g} / \mathrm{km}$ & $\mathrm{CO} / \mathrm{g} / \mathrm{km}$ & $\mathrm{NOx} / \mathrm{g} / \mathrm{km}$ & $\mathrm{FC} / \mathrm{L} / 100 \mathrm{~km}$ \\
\hline $\begin{array}{c}\text { Before } \\
\text { optimization }\end{array}$ & 0.546 & 2.146 & 0.252 & 4.745 \\
\hline $\begin{array}{c}\text { After } \\
\text { optimization }\end{array}$ & 0.532 & 2.013 & 0.131 & 2.833 \\
\hline $\begin{array}{c}\text { Control } \\
\text { strategy }\end{array}$ & $\mathrm{HC} / \mathrm{g} / \mathrm{km}$ & $\mathrm{CO} / \mathrm{g} / \mathrm{km}$ & $\mathrm{NOx} / \mathrm{g} / \mathrm{km}$ & $\mathrm{FC} / \mathrm{L} / 100 \mathrm{~km}$ \\
\hline $\begin{array}{c}\text { Before } \\
\text { optimization }\end{array}$ & 0.466 & 2.113 & 0.263 & 5.084 \\
& & & & \\
\end{tabular}

\begin{tabular}{ccccc}
\hline $\begin{array}{c}\text { After } \\
\text { optimization }\end{array}$ & 0.428 & 2.021 & 0.174 & 3.125 \\
\hline \multirow{4}{*}{$\begin{array}{c}\text { Control } \\
\text { strategy }\end{array}$} & $\mathrm{HC} / \mathrm{g} / \mathrm{km}$ & $\mathrm{CO} / \mathrm{g} / \mathrm{km}$ & $\mathrm{NOx} / \mathrm{g} / \mathrm{km}$ & $\mathrm{FC} / \mathrm{L} / 100 \mathrm{~km}$ \\
\hline $\begin{array}{c}\text { Before } \\
\text { optimization }\end{array}$ & 1.087 & 5.391 & 0.289 & 4.145 \\
\hline $\begin{array}{c}\text { After } \\
\text { optimization }\end{array}$ & 1.055 & 5.248 & 0.176 & 2.336 \\
\hline
\end{tabular}

As it can be see from table1: the optimized fuzzy control strategy has greatly improved the fuel economy and decreased all emission index significantly, ensure the battery SOC change in the small scope to to extend its service life, figure 3 shows the changes of the battery SOC in CYC_NEDC.

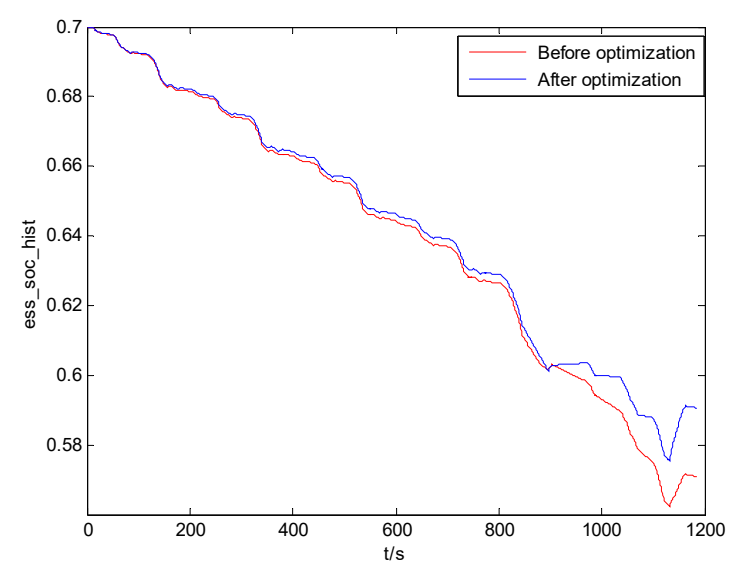

Fig. 3. Comparison of battery SOC in CYC_NEDC

\section{Conclusion}

Aiming at parallel hybrid electric vehicle, this paper designed a fuzzy controller by using vehicle torque request and battery SOC as inputs, engine torque as output, proposed to use improved particle swarm algorithm which based on dynamic learning factor and adaptive inertia weight to optimize it, simulation results show that the optimized fuzzy control effectively improved the fuel economy and emissions of the vehicle, and maintain the SOC balancee.

\section{References}

1. Fu Zhumu, Wang Conghui and $\mathrm{Pu}$ Jiexin. Modeling and Simulation of Fuzzy Control Energy 
Management Strategy for PHEV [J]. Journal of System Simulation,2012, 24(6): 1314-1319.

2. CHAN C C, ALAIN B, CHEN K Y. Electric, hybrid, and fuel-cell vehicles: architectures and modeling [J]. IEEE Transactions on Vehicular Technology, 2010,59(2): 589-598.

3. Wu Jian, Zhang Chenghui. Fuzzy energy management strategy of parallel hybrid electric vehicle based on particle swarm optimization[J]. Control and Decision, 2008, 23(1):46-50.

4. Cui Ting, Sun Yuanzhang, Xu Jian, et al. Reactive power optimization of power system based on improved niche genetic algorithm [J]. Proceeding of the CSEE,2011,31(9):43-50(in Chinese).

5. ANTONIA S, LINNO G. Control of hybrid electric vehicles [J]. IEEE Control Systems Magazine, 2007, 26(4):61-70. 